\title{
Development of wireless charging system along with power line communication used in Electric Vehicles
}

\author{
Sai Varun Alapati ${ }^{1 *}$, Indusaiteja Nadella $^{2 *}$, Phaneendra Babu Bobba $^{3}$ and Madhur Deo Upadhayay ${ }^{4}$ \\ ${ }^{1}$ Dept of Computer Science,State University of NewYork at Buffalo, Buffalo, New York, USA \\ ${ }^{2}$ Senior Software Developer Associate, NTT Data GDS PVT Ltd, Hyderabad, Telengana, India \\ ${ }^{3}$ SMIEEE, Dept. of EEE, GRIET, Hyderabad, Telangana, India \\ ${ }^{4}$ Electrical Engineering Department Shiv Nadar University, Greater Noida, India
}

\begin{abstract}
Charging an Electric Vehicle wirelessly is the latest technology being developed for the electric vehicles replacing the traditional way of plugging to the supply. In this paper, authors explained how to integrate Power Line Communication along with wireless power transfer in EV. The entire system is implemented in ADS simulation software. We are adapting to magnetic resonance coupling method for wireless power transfer in EV. The overall ideology of the project is to design an innovative system which involves higher power transfer and implement smart communication system between vehicle and the grid while following the latest magnetic resonance charging standards.,
\end{abstract}

Keywords Electric Vehicle (EV), Power Line Communication (PLC), wireless power transfer, communication protocol, Wireless Power Transfer (WPT), Power Transfer Efficiency (PTE)

\section{Introduction}

The cost of fuels like petrol, diesel etc. has been steadily increasing due to increased number of vehicles and proportional excess usage of fuel. Depleting sources of these fuels are also a major concern. These age old designs of vehicles are the major contributors to the problem of greenhouse gases.

The future of automotive technologies is moving toward electric vehicles which are considered as a replacement to oil-powered internal combustion engine driven vehicles, keeping in mind $\mathrm{CO} 2$ reduction. Alternate energy Plug-in Electric Vehicles (PEVs) have been proposed to achieve environment friendly transportation. Even though the PEV usage is currently increasing, a breakthrough would be required to overcome battery related drawbacks.

Furthermore, PEVs have not been accepted as a preferred choice by many consumers due to charging related issues. This is the major issue faced in the field currently.

Wireless power transfer (WPT) was discovered and enacted by Nikola Tesla. This was further enhanced by many other scientists. The concept has been

* Corresponding author: saivarun@buffalo.edu

* Corresponding author: Indusaiteja.nadella@gmailcom implemented in many applications for e.g. mobile charging, laptop charging, and electric vehicles etc. However application in electric vehicles was a major finding in the field of Automobile and Transportation. Wireless power transfer methods are bounded with technologies such as Radio waves, micro waves, photoelectric, laser, inductive coupling and magnetic resonance coupling. Based on the power transfer distance WPT methods are categorized into near field and far field. Inductive coupling and magnetic resonance coupling are regarded as near field transfer methods; whereas the other methods are categorized into far field approach. The magnetic resonance coupling method is recommended for WPT of EV because of high range and higher frequencies secured by the method when in compared with other WPT methods.

In [1], Chun et al. discuss about the various methods in WPT for EV charging. Out of which the strongly recommended one is the coupled resonance method for usage in EV's. The challenges faced in any method are the coil design, as they decide upon the upper boundary limit of power capacity and also the efficiency which depends on the quality factor $(\mathrm{Q})$ of the coils. The frequency range at which the coil is designed varies from $10 \mathrm{kHz}$ to $150 \mathrm{kHz}$ and even more, but we obtain high ac 
resistance when there is an increase in frequency which leads to decrease of skin effect. The high ac resistance is also observed due to proximity effect as the eddy currents are flown along the strands of the wire.

In [4], Miller et al. provides an explanation to use litz wires so as to reduce the ac resistance. It reduces the effect of both skin and proximity effects by ensuring the ac current to flow uniformly throughout the wire. Smaller diameters of copper wires are used so that radius of each individual strand is less than their skin depth, so the higher the frequency the smaller the inductor. Litz wire also increases the range of frequencies which can be used, that give high efficiency and whose weight is very less when in compared with conventional wire.

In [5], Subhadeep et al. experiment in comparing coil structures among circular spiral, square spiral and helical coils. Out of which the inductance is observed to be least for circular spiral coil and highest for helical shaped coil. This paper also proposes a multilayer spiral structure which observes high $Q$ factor when it had necessary space constraint and a better field distribution in coils.

As the coil design is important, in [3] Rikard et al. uses a circular spiral coil which at a source frequency of $85 \mathrm{kHz}$ is supplied and both the coils are identical and shielding is done with a ferrite core, where the coils are places at $30 \mathrm{~cm}$ apart and checked the shielding effect on vehicle side and ground side both inclusive and also placing shielding only on vehicle side. The WPT's develop a lot of magnetic fields as the power is transferred wirelessly but they are ensured that they are safe to use by the general public keeping in mind about the health of humans as well as animals. The power in reference [3] is transferred at a peak value of $1.3 \mathrm{~kW}$ with an efficiency of $90 \%$, air gap maintained at $0.3 \mathrm{~m}$.

As WPT is hazardous for humans as well as animals, we must ensure that certain standards are to be followed while implementing WPT in the system. In [6], author discuss mainly of standards being followed in US and Europe. In US, the main standard for EV charging is the Society of Automotive Engineers (SAE) standard J1772. Whereas in Europe the standard followed is International Electro technical commission (IEC) 61851. The above mentioned standards define the requirements for "Electric Vehicle Supply Equipment" (EVSE).

In [7] the discussion is about SAE TIR J2954 standard which establishes an industry-wide specification guideline that defines acceptable criteria for interoperability, electromagnetic compatibility, minimum performance, safety and testing for wireless charging of light duty electric and plug-in electric vehicles. The standard SAE TIR J2954 is intended for stationary applications (charging while vehicle is not in motion).

Based on [3], the study about the WPT system and coil design, the circular spiral coil is taken in consideration with [3] as the required power rating for the application is near about $1.3 \mathrm{~kW}$. Thus, we are implementing magnetic resonance method for WPT in our system.

The development of Li-ion battery packages show the need of a Battery Management System (BMS) which improves the performances of all cells ensuring correct charging/discharging, continuous monitoring for preventive maintenance etc. A BMS needs data transfer, which can be efficiently performed by the use of a PLC (Power Line Communications) data channel demonstrated in [8]

In [9], the Qi standard demonstrates that the communication between Transmitter and Receiver happens in the form of packets. A Packet consists of a preamble, a header byte, a message, and a checksum. A Packet is named after the kind of message that it contains.

In [10], authors proposed a magnetically coupled resonator composed by four resonant circuits, magnetically linked by coupling coefficients and simulated the WPT channel frequency response. They calculated the channel capacity for a WPT system by using data communication transfer as a function of the coupling coefficient, $\mathrm{k}$ and by considering its frequency response and additive white Gaussian noise (AWGN). They proposed architecture for integrating the PLC in a WPT system.

In [11], author proposed a coupled WPT-PLC as a continuation to [10] and constructed a two coil system and measured the scattering parameters used to design a four port system and implemented a simulation package in ADS. Their results confirmed the theoretical studies previously published and showing that the system guarantees power transfer together with reasonable and effective data transfer.

\section{Wireless Power Transfer in Electric Vehicles}

\subsection{MAGNETIC RESONANCE COUPLING}

Magnetic Resonance is a near field WPT technology. It works on the principle of resonance of inductance and capacitance present in the circuit. If two or more pairs of RLC resonators are used then the WPT system is used to increase the PTE and extend its range. The inductive power transfer and magnetic resonance coupling come under near field WPT technologies, but there is a small difference between these two technologies. The frequency range at which the power transfer can be done is higher in magnetic resonance coupling when in compared with inductive coupling. With the usage of more pairs of RLC's the coupling becomes stronger and is known as strongly coupled resonance circuit as mentioned in [1]. We use an equivalent circuit theory method (Thevenin equivalent) to find the circuit parameters such as currents and voltage across coils, 
compensation capacitors. So it is essential to use Thevenin equivalent method to estimate component ratings in designing high power WPT system. The two coil systems consists of a primary coil and secondary coil whereas the four coil system consists of two extra coils known to be drive loop and load loop which are coupled with transmitter coil and receiver coil respectively. Both the coil systems have their own advantages and disadvantages. But we consider two coil systems for our project and proceed per [2].

\subsection{EV CHARGING SCHEMES}

Electric vehicles are successful only when the charging stations are easily accessible to the public when required. The critical point to think is about how are the grids in support with the electric vehicle specifications.

The first place considered for charging stations should be homes and workplaces. Other potential locations with high populations include gas stations, shopping centres, restaurants, entertaining places, highway rest areas, municipal facilities, and schools.

There have been various standards regarding the energy transfer, connection interface and communication for EV charging, the following table summarizes the standards to be followed for Electric vehicle charging schemes [2]:

Table 1. STANDARDS OF EV CHARGING

\begin{tabular}{|l|l|}
\hline Standard & Title/description \\
\hline $\begin{array}{l}\text { National } \\
\text { Electric } \\
\text { Code }\end{array}$ & Electric Vehicle Charging System \\
\hline SAE J2293 & Energy Transfer System for Electric Vehicles \\
\hline SAE J2836 & $\begin{array}{l}\text { Recommended Practice for Communication } \\
\text { between Plug-in and Vehicles and Utility Grid }\end{array}$ \\
\hline SAE J1772 & Electric Vehicle Conductive Charge Coupler \\
\hline SAE J1773 & Electric Vehicle Inductively Charge Coupler \\
\hline IEC 62196 & $\begin{array}{l}\text { Plugs, socket outlets, vehicle couplers and vehicle } \\
\text { inlets-Conductive charging of electric vehicles }\end{array}$ \\
\hline $\begin{array}{l}\text { IEEE } \\
\text { 1547.3 }\end{array}$ & $\begin{array}{l}\text { Interconnecting Distributed Resources with } \\
\text { Electric Power Systems }\end{array}$ \\
\hline
\end{tabular}

\section{Methodology for PLC Implementation}

In the scheme shown in the Fig. 1 the power signal and the data signal are superimposed to form a PLC signal at the outlet. There are two tasks of this WPT system. First, transmitting the power from the power source to the load and enabling the communication to the PLC Device. Second, the high power signal at low frequency is separated from the high frequency, low power PLC signal, by using a low pass and a high pass filter respectively. By using a DC to radio frequency amplifier, the power signal is then transformed to DC and then converted to a high power sinusoid at the resonance frequency $f_{O}$ of the WPT system. In order to remove the out of band noise generated by the amplifier, the output of the RF amplifier is filtered by a band pass

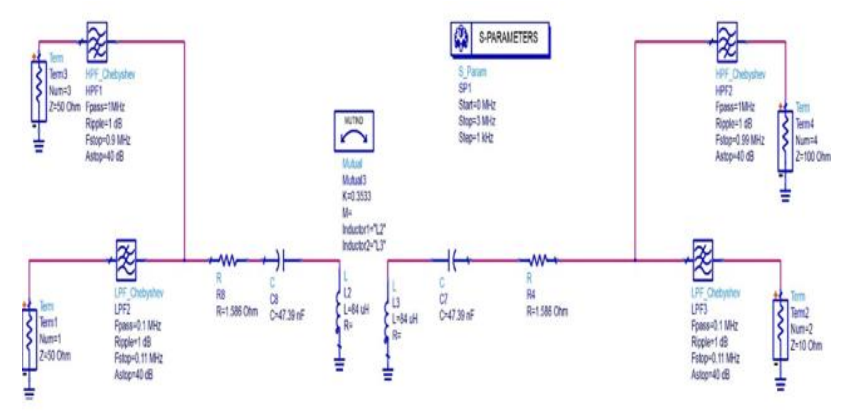

Fig.2. Proposed WPT-PLC system

filter centered at fo. The PLC signal is coupled at the exit stage of the amplifier and to prevent the propagation of high power signal generated by the DC/RF amplifier through the PLC signal path, a band stop filter with a narrow stop-band centered at fo is added. The voltage source, $\mathrm{V}_{\text {source }}$ at this point i.e., the input of the drive loop is represented by the sum of the high power radiofrequency signal centered in fo and the low power PLC signal. This signal then propagates to the load loop through the WPT resonators. At the output of the load loop, a band pass filter centered at $\mathrm{f0}$ extracts the power signal and sends it to a $\mathrm{RF} / \mathrm{DC}$ rectifier to provide the power to the DC load. A band stop filter centered at $\mathrm{f} 0$ is used to remove the power signal from the superimposed signal to get PLC signal, which is then coupled to a PLC device.

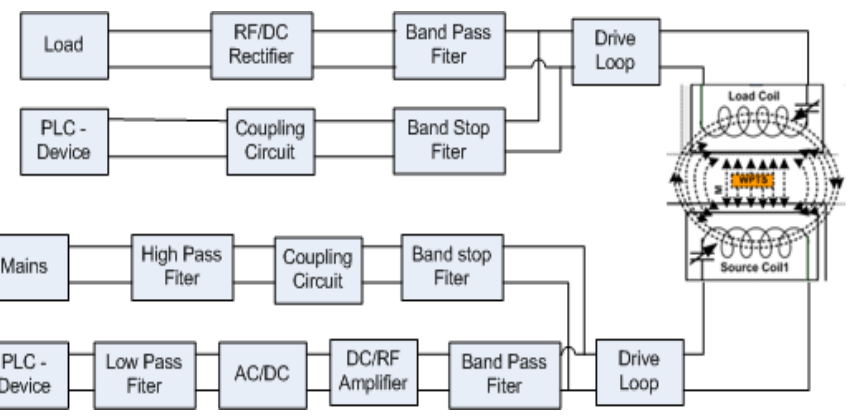

Fig.1. PLC and Implementation of system block diagram

In the above scheme, the power transmission is unidirectional where as it allows a bidirectional communication. The PLC device connected to the load loop can be enabled to transmit data that will propagate in the same method from the receiver coil to the transmitter coil as a low power broadband signal. The WPT systems are mainly designed to have two resonance frequencies one peak for power transfer. So we can use the frequencies around the other peak for the PLC communication.

\section{Simulation results}

\subsection{Proposed WPT-PLC system}


The scheme of the coupled system is shown in Fig.2, which is implemented in circuit simulator ADS. Port 1, port 2 are the input and output terminations for the power channel and port 3, port 4 are the input and output terminations for the power line data signal.

The power signal is transmitted through the coupling inductors and decided to adopt the power transmission frequency of $85 \mathrm{kHz}$ and the communication signal is transmitted through the band $1-3 \mathrm{MHz}$. The are two $1 \mathrm{MHz}$ chebyshev high-pass filters and the two $0.1 \mathrm{MHz}$ chebyshev low-pass filters of band pass with a ripple of $1 \mathrm{~dB}$ and an attenuation for all the filters in order to isolate the data from the power channels and vice-versa.

Fig.3 represents frequency response of the transmission coefficients $\mathrm{S}(2,1)$ (in terms of the scattering matrix) on the power channel at $\mathrm{K}=0.35$ with peak value 0.82 . The frequency chosen for the power link is $85 \mathrm{kHz}$.

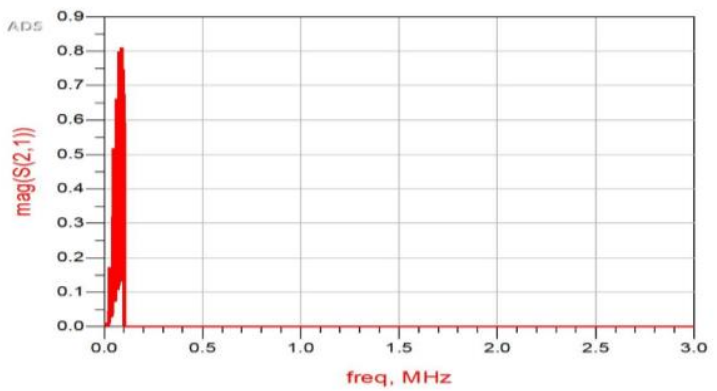

Fig.3. Transmission coefficient $\mathrm{S}(2,1)$ on the Power Channel at $\mathrm{K}=0.35$

Fig.4, represents frequency response of the transmission coefficients $\mathrm{S}(2,1)$ (in terms of the scattering matrix) on the power channel at $\mathrm{K}=0.6$ with peak value 0.90 . The frequency chosen for the power link is $85 \mathrm{kHz}$.

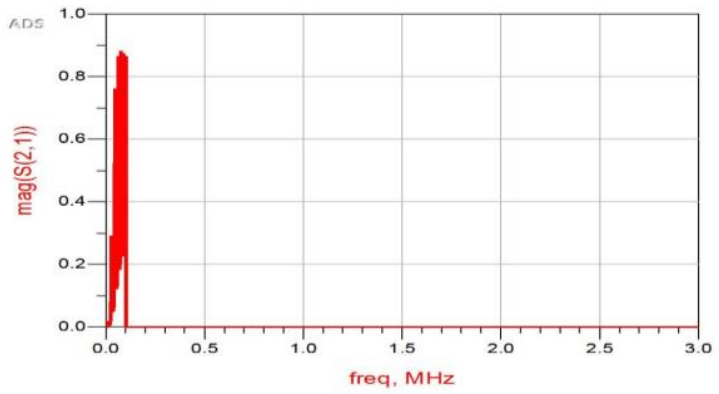

Fig.4 Transmission coefficient $\mathrm{S}(2,1)$ on the Power Channel at $\mathrm{K}=0.6$.

Fig.5 represents frequency response of the reflection coefficients $\mathrm{S}(1,1)$ (in terms of the scattering matrix) on the power channel with $\mathrm{K}=0.35$. The frequency chosen for the power link is $85 \mathrm{kHz}$.

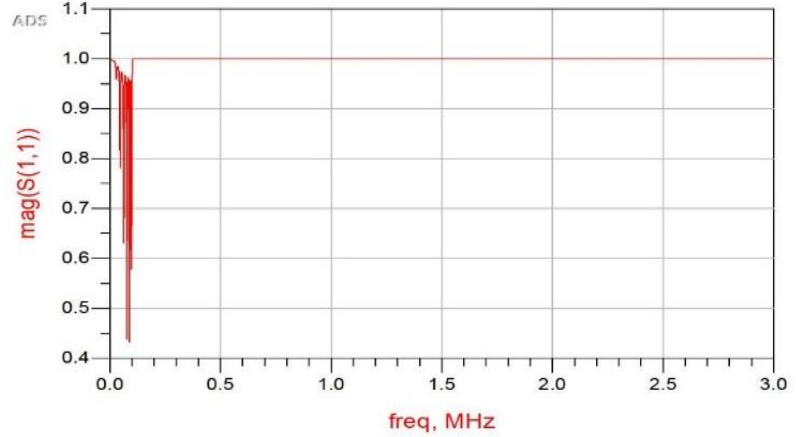

Fig.5. Reflection coefficient S $(1,1)$ on the Power Channel

Fig.6 represents frequency response of the reflection coefficients $S(1,1)$ (in terms of the scattering matrix) on the power channel with $\mathrm{K}=0.6$. The frequency chosen for the power link is $85 \mathrm{kHz}$.

Fig.6. Reflection coefficient $\mathrm{S}(1,1)$ on the Power Channel at $\mathrm{K}=0.6$

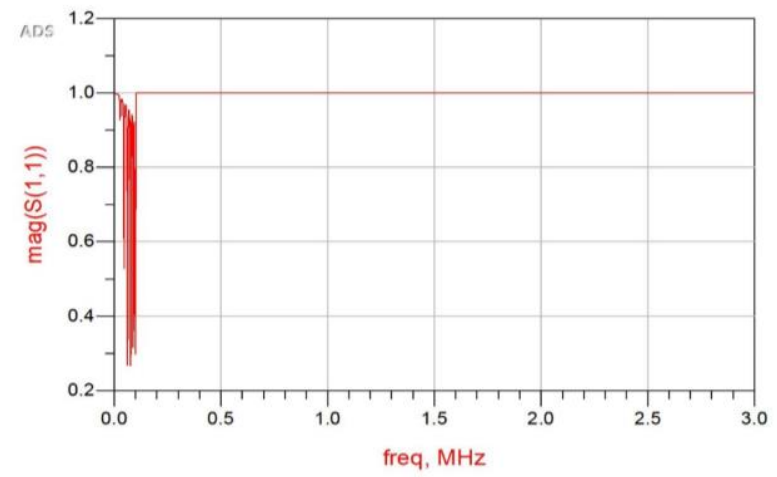

Fig.7 represents frequency response of the transmission coefficient $\mathrm{S}(4,3)$ (in terms of the scattering matrix) on the Power line channel in the frequency range $1-3 \mathrm{Mhz}$ with $\mathrm{K}=0.35$. The frequency chosen for the power link is $85 \mathrm{kHz}$.

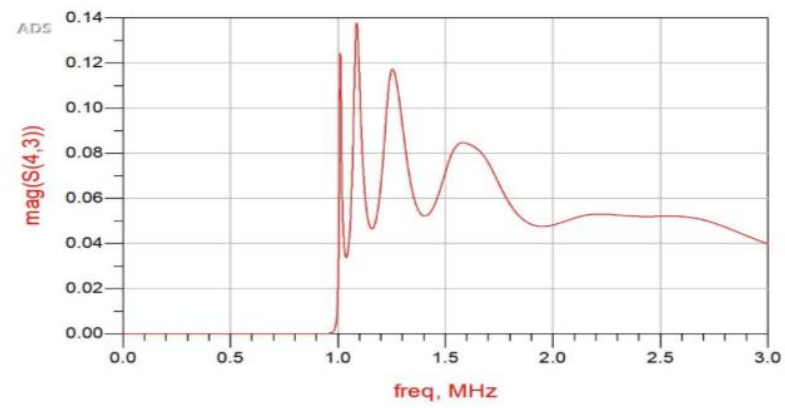

Fig.7. Transmission coefficient $\mathrm{S}(4,3)$ on Power Line Channel in the frequency range 1-3 MHz with $\mathrm{k}=0.35$.

Fig.8 represents frequency response of the transmission coefficient $\mathrm{S}(4,3)$ (in terms of the scattering matrix) on the Power line channel in the frequency range $1-3 \mathrm{MHz}$ with $\mathrm{K}=0.6$. The frequency chosen for the power link is $85 \mathrm{kHz}$. 


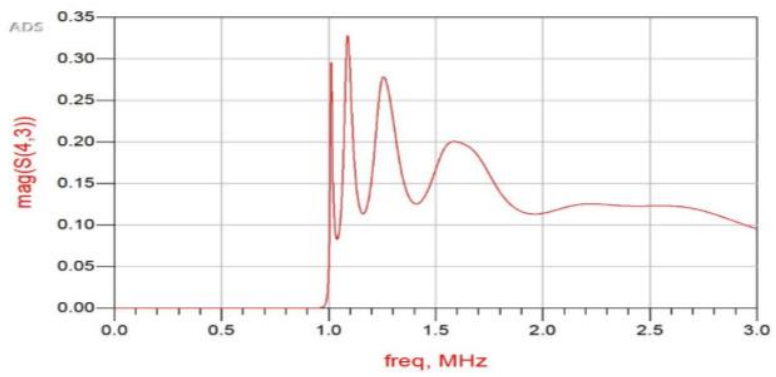

Fig.8. Transmission coefficient $S(4,3)$ on Power Line Channel in the frequency range $1-3 \mathrm{MHz}$ with $\mathrm{K}=0.6$.

Fig.9 shows the isolation between the input port of the Power channel and the input/output ports of the power line data channel at $\mathrm{K}=0.35$. The frequency chosen for the power link is $85 \mathrm{kHz}$.

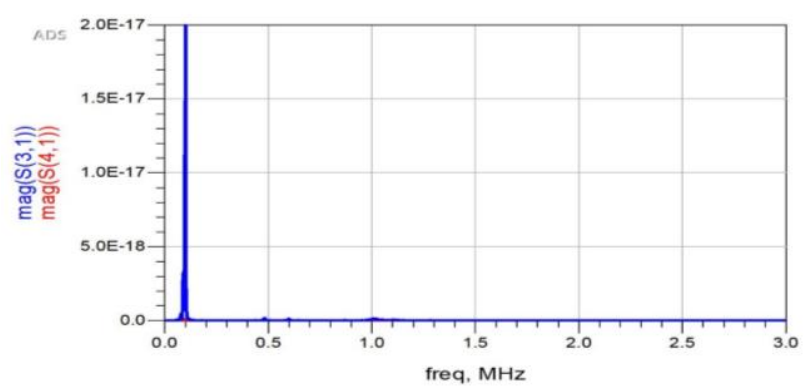

Fig.9. Isolation among the input port of the Power channel and the input (3) and output (4) ports of the Power Line Data channel at $\mathrm{K}=0.35$.

Fig.10 shows the isolation between the input port of the Power channel and the input/output ports of the power line data channel at $\mathrm{K}=0.6$. The frequency chosen for the power link is $85 \mathrm{kHz}$.

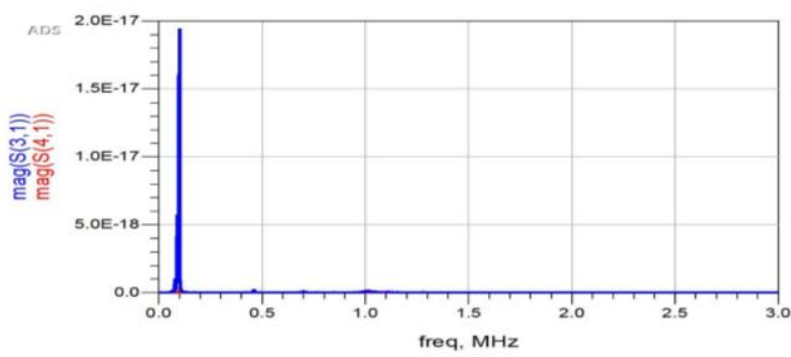

Fig.10. Isolation among the input port of the Power channel and the input (3) and output (4) ports of the Power Line Data channel at $\mathrm{K}=0.6$.

\section{Conclusions}

In this paper authors implemented magnetic resonance coupling method for wireless power transfer in EV and also demonstrated how to integrate Power Line Communication along with wireless power transfer in EV's. The simulation results demonstrates that the transmission coefficient of the power signal is acceptable at $\mathrm{k}=0.35$ and as we increase $\mathrm{k}$ to 0.6 the transmission coefficient increases, while both $\mathrm{k}$ values guarantees power transfer together with reasonable and effective data transfer. The power signal can be improved by increasing the power transmission frequency with permissible $\mathrm{k}$.

\section{References}

1. Chun Qiu, K.T. Chau, Chunhua Liu, and C.C. Chan, "Overview of Wireless Power Transfer for Electric Vehicle Charging", in EVS27 International Battery, Hybrid and Fuel Cell Electric Vehicle Symposium, Barcelona, Spain, November 17-20, 2013, pp. 1-9.

2. D.M. Vilathgamuwa and J.P.K. Sampath, "Wireless Power Transfer (WPT) for Electric Vehicles (EVs) Present and Future Trends", in Plug In Electric Vehicles in Smart Grids, S. F. Rajakaruna S., Ghosh A., Ed., ed Springer International Publishing AG, Part of Springer Science+Business Media: Springer Singapore, 2015, pp. 33-60.

3. Rikard Vinge, "Wireless Energy Transfer by Resonant Inductive Coupling", , Master of Science Thesis, Department of Signals and systems, CHALMERS UNIVERSITY OF TECHNOLOGY, Goteborg, Sweden 2015.

4. J.M. Miller, C.P. White, O.C. Onar, and P.M. Ryan, "Grid side regulation of wireless power charging of plug-in electric vehicles," in Proc. Energy Conv. Congress Expo. Raleigh, NC, 2012, pp. 261-268.

5. Subhadeep Bhattacharya, "Design of Static Wireless Charging Coils for Integration into Electric Vehicle", IEEE ICSET 2012, Nepal, pp. 146 - 151.

6. WiTricity, WiT-3300, [Online]. Available: http://witricity.com/innovation/standards/

7. SAE International, Hybrid-Ev committee [Online]. Available: http://standards.sae.org/wip/j2954/

8. I. Ouannes, P. Nickel, and K. Dostert, "Cell-wise monitoring of lithiumion batteries for automotive traction applications by using power line communication: battery modeling and channel characterization," in Proc. 18th IEEE Int. Symp. on Power Line Commun. and its Appl., Mar. 2014, pp. 24-29

9. "http://www.qiwireless.com/category/wireless-standards/" QIWireless

10. S. Barmada, M. Tucci, M. Raugi "Power Line Communication Integrated in a Wireless Power Transfer System: a Feasibility Study",Proceedings of International Symposium on Power-Line Communications and Its Applications, ISPLC 2014, 30 March - 2 April, 2014, Glasgow, Scotland, pp. 116-120.

11. S. Barmada, M. Tucci,M. Raugi M. Dionigi, P. Mezzanotte "Experimental Validation of a Hybrid Wireless Power Transfer - Power Line Communication System" International Symposium on Power Line Communications and its Applications (ISPLC), 20 